\title{
Status of the Large Hadron Collider (LHC)
}

\author{
Lyndon R. Evans
}

\begin{abstract}
The Large Hadron Collider (LHC), due to be commissioned in $\mathbf{2 0 0 7}$, will provide particle physics with the first laboratory tool to access the energy frontier above $1 \mathrm{TeV}$. In order to achieve this, protons must be accelerated and stored at $7 \mathrm{TeV}$, colliding with an unprecedented luminosity of $10^{34} \mathrm{~cm}^{-2} \mathrm{~s}^{-1}$. The 8.3 tesla guide field is obtained using conventional NbTi technology cooled to below the lambda point of helium. The machine is now well into its installation phase, with first beam injection foreseen for spring 2007. A brief status report is given and future prospects are discussed.
\end{abstract}

Index Terms-Accelerators, cryogenics, superconductors.

\section{INTRODUCTION}

$\mathbf{T}$ HE LHC machine is now in its installation phase. The tunnel infrastructure is being prepared and the installation of the critical cryogenic distribution line has started. Installation of the magnetic elements in the TI8 transfer line is underway and installation of superconducting magnets in the first sector of the ring itself will start in early 2004. A major milestone will be the injection and transport of a beam around the first octant of the machine in April 2006. Commissioning of major hardware items, including the four new $18 \mathrm{~kW}$ cryogenic plants is already underway.

Considerable progress has been made in preparing the beam for operation of the LHC at nominal luminosity in the injector chain. In the PS, a novel method of producing the required $25 \mathrm{nsec}$ bunch structure has been successfully applied. In the SPS, the predicted electron cloud instability has clearly been observed and the required conditioning of the vacuum chamber by the beam itself (scrubbing) has been achieved.

Production of the most critical components has now ramped up to plateau and has allowed a reliable schedule for machine commissioning and operation to be defined in collaboration with the spokesmen for the four large detectors. The ring will be closed by the end of 2006 and the injection of the first beams is foreseen in April 2007.

\section{INJECTORS}

The existing injector chain will be used for producing the LHC beams. In order to achieve the required performance in terms of bunch intensity and emittance with the nominal bunch spacing of $25 \mathrm{~ns}$, a number of modifications are required, particularly in the PS complex. The classical technique of generating the $25 \mathrm{~ns}$ bunch spacing $(\mathrm{h}=84$ in the PS) from the beam injected from the PS Booster $(\mathrm{h}=7)$ is by debunching and rebunching on the higher harmonic. This technique suffers

Manuscript received October 20, 2003.

The author is with LHC project Leader, CERN, 1211 Geneva, 23, Switzerland (e-mail: Lyn.Evans@cern.ch).

Digital Object Identifier 10.1109/TASC.2004.829030

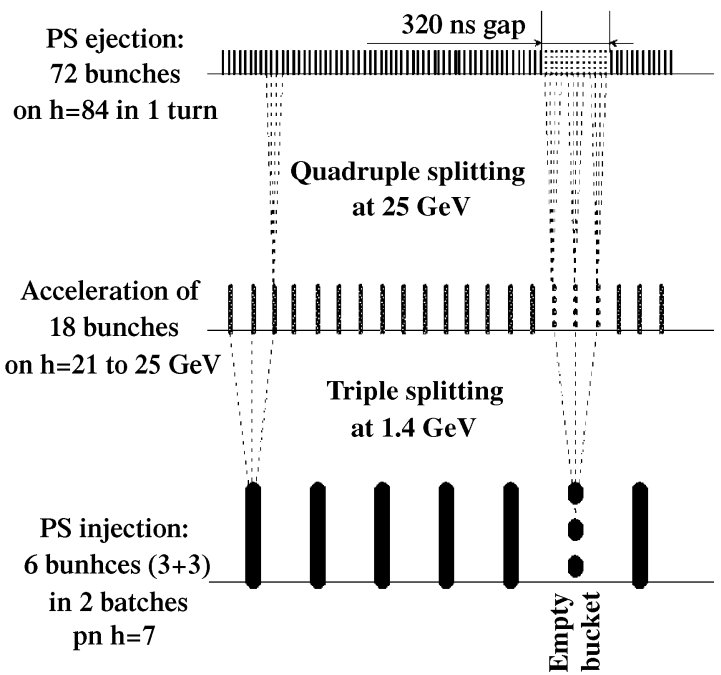

Fig. 1. PS gymnastics for the $25 \mathrm{~ns}$ bunch train.

from two major disadvantages. The $25 \mathrm{~ns}$ spacing is too small for the risetime of the extraction kickers, resulting in a number of bunches lost or with large betatron oscillations. In addition, the beam tends to become unstable during the debunching process when the energy spread becomes too low for adequate Landau damping. To overcome these problems, a novel technique has been developed [1] which consists of stepwise bunch splitting using intermediate harmonic cavities. By leaving out one Booster bunch (Fig. 1), a large enough gap can be left for the kickers and the beam never gets unstable since the energy spread is too large. Using this technique, beams satisfying the LHC specifications on emittance, intensity and bunch length have already been obtained.

This beam is now available for commissioning the next link in the injector chain, the SPS. Machine studies in the SPS have already clearly demonstrated the onset of the electron cloud instability (see below) as predicted. The benefit of the impedance reduction campaign carried out over the last three years in the SPS has now become clear, [2] with an important improvement in beam stability and a reduction of the longitudinal impedance of the beam for the LHC.

\section{MAGNETS}

The LHC will require more than 8000 superconducting magnets of different types. The most challenging are the 1232 superconducting two-in-one dipoles, which must operate reliably at the nominal field of 8.3 tesla corresponding to a center-of-mass energy of $14 \mathrm{TeV}$, with the possibility of being pushed to an ultimate field of 9 tesla.

Production of the critical superconducting cable is now at plateau, with about half of the required quantity produced 


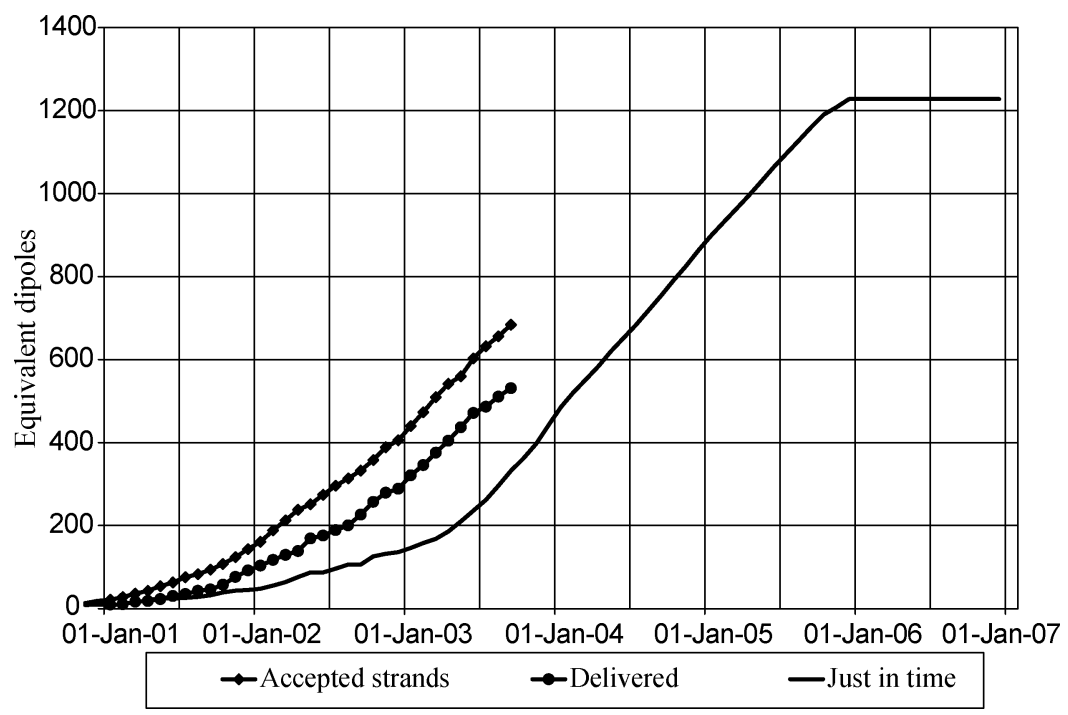

Fig. 2. Production of LHC outer layer superconducting strand and cable. The full line shows the just-in-time line for dipole manufacture.

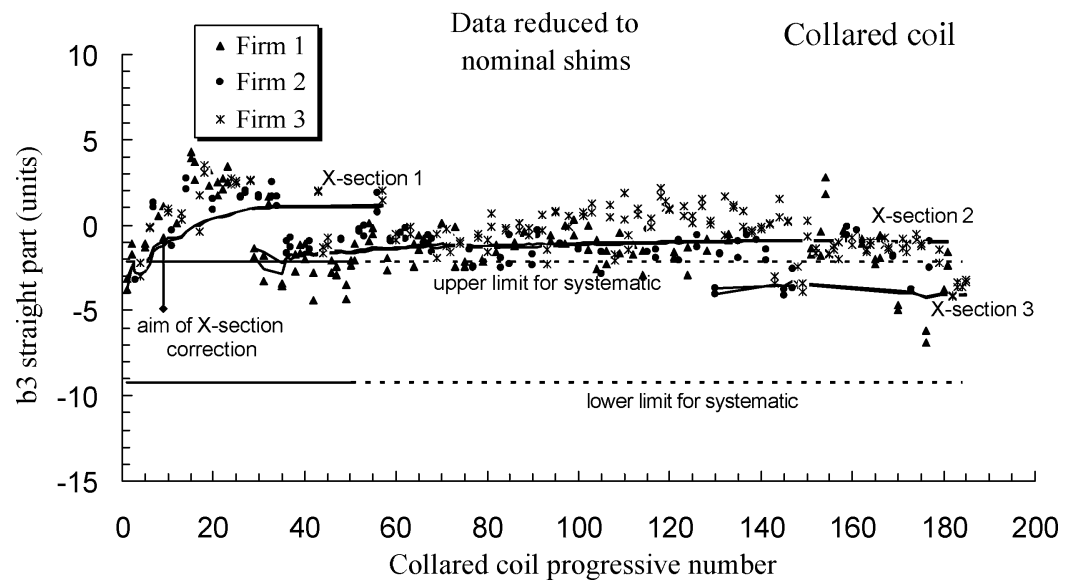

Fig. 3. Evolution of regular sextupole $\left(b_{3}\right)$ in the main dipoles. A correction was made after the first ten dipoles. A second correction was made after production of the first octant.

(Fig. 2). Dipole manufacture is ramping up according to schedule. More than 100 dipoles have been delivered and are in the process of cold testing. The field quality is being carefully monitored by warm magnetic measurements on collared coils in the factories. At the beginning of production, it was necessary to make a small modification of the coil cross section to reduce the normal sextupole component [3]. Production has continued with this cross section, which is acceptable but with b3 at the high end of the tolerance window (Fig. 3), for the whole of the first octant. Recently a further small correction has been made to move $\mathrm{b} 3$ and $\mathrm{b} 5$ well inside the window, making production less vulnerable to upward drift in the future.

Over 60 dipoles have been cold tested. They reach nominal field after a few settling quenches (Fig. 4) and are then systematically ramped up to $9 \mathrm{~T}$. A few dipoles have been rejected for bad performance and are being reworked.

The 400 main quadrupoles are integrated into Short Straight Sections (SSS) also containing sextupole and dipole for chromaticity and orbit correction respectively. The quadrupoles have been designed at Saclay in the framework of a collaboration with CEA. Series production has now started with the first quadrupoles tested falling well within the specification. Fig. 5 shows the first series SSS on its cold measurement bench.

The dispersion suppressors, long straight sections and insertion regions contain many special magnets, the most challenging of which are the high gradient $(220 \mathrm{~T} / \mathrm{m})$ large aperture $(70 \mathrm{~mm})$ quadrupoles for the inner triplets of the low-beta insertions. Two different versions of these quadrupoles have been designed at FNAL and KEK, and have been validated through short models. Series production of the Japanese magnets is now almost complete and the production of the US units is about half way complete.

Each insertion triplet will contain a mixture of US and Japanese magnets. The final integration into the cryostats is done at FNAL in collaboration with LBL.

Many other superconducting magnets are needed for trimming the optics and for correcting lattice imperfections. A full list is given in Table I.

Contracts for all elements have been placed in industry and series production has started. Half of the MCS and MCDO magnets are being manufactured in India in the framework of a collaboration agreement. Detailed reports on the LHC supercon- 


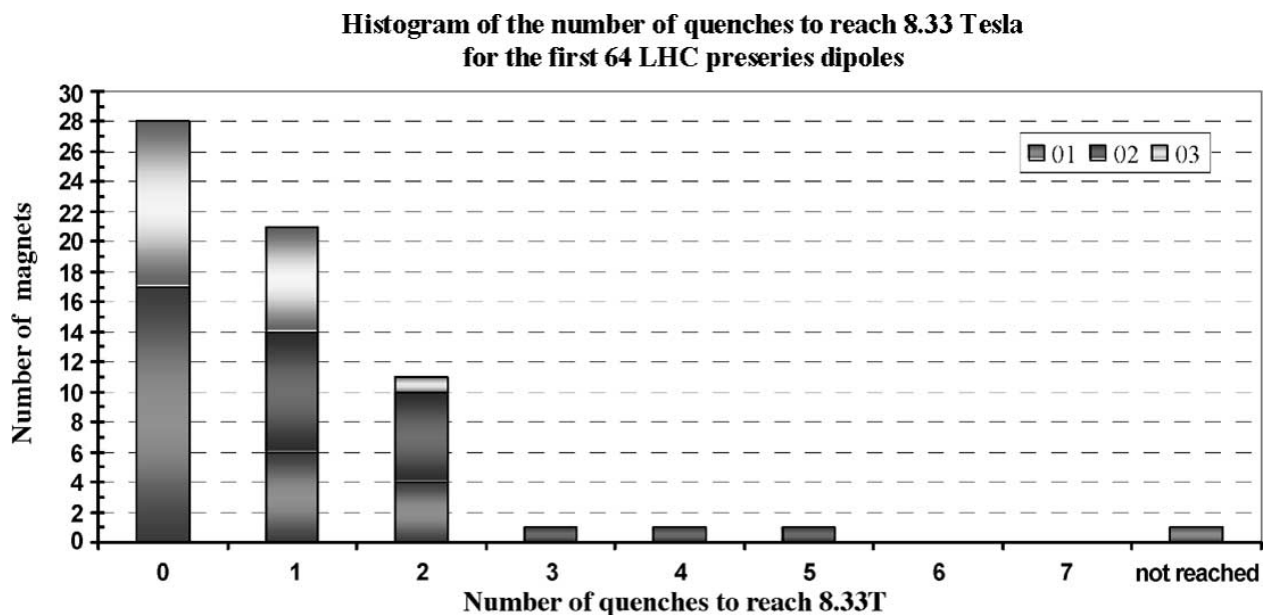

Fig. 4. Histogram of the number of quenches to nominal field for the first 64 dipoles.

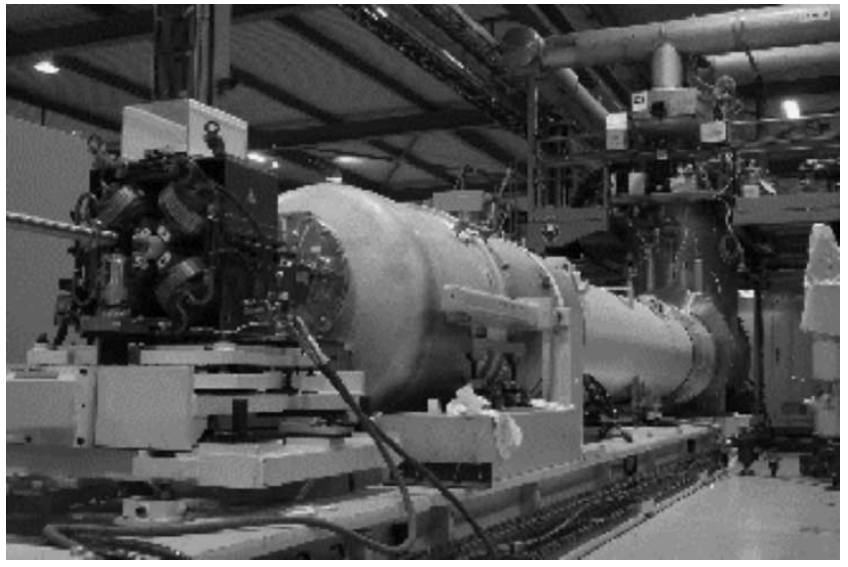

Fig. 5. The first series short straight section on its test stand.

\section{TABLE I}

\begin{tabular}{|c|c|c|}
\hline Name & Quantity & Purpose \\
\hline MB & 1232 & Main dipoles \\
\hline MQ & 392 & Main quadrupoles \\
\hline MSCB & 376 & Combined chromaticity/ closed orbit correctors \\
\hline MCS & 2464 & $\begin{array}{l}\text { Dipole spool sextupole for persistent currents at } \\
\text { injection }\end{array}$ \\
\hline MCDO & 1232 & Dipole spool octupole/decapole for persistent currents \\
\hline $\mathrm{MO}$ & 336 & Landau octupole for instability control \\
\hline MQT & 256 & Trim quad for lattice correction \\
\hline MCB & 266 & Orbit correction dipoles \\
\hline MQM & 100 & Dispersion suppressor quadrupoles \\
\hline MQY & 20 & Enlarged aperture quadrupoles \\
\hline
\end{tabular}

ducting magnet status can be found in specialized contributions to this conference [4]-[6].

A large number of conventional magnets are also required. In the two transfer lines from the SPS to LHC there are 360 six meter long dipoles and 180 quadrupoles, all manufactured at BINP Novosibirsk in the framework of the CERN/Russia agreement. The delivery of these magnets is now complete. Also from Russia are the warm separation dipoles (BINP) and the septum magnets for the beam abort channels (IHEP, Protvino).
Two of the long straight sections of the LHC are used for beam collimation. In view of the high radiation levels these straight sections are equipped with special twin aperture warm quadrupoles (52 units) built in Canada in the framework of the CERN/TRIUMF agreement. Delivery of these magnets is now complete.

\section{CRYOGENICS}

The most important elements of the cryogenic system are the eight large $(18 \mathrm{~kW})$ cryoplants together with their associated $1.8 \mathrm{~K}$ refrigeration units for the production of superfluid helium, and the cryogenic distribution line feeding the magnet system. Four of the $18 \mathrm{~kW}$ plants will be recuperated from the LEP cryogenic system with suitable upgrades. All four of the new plants have now arrived at CERN, three have been successfully accepted and the last one is being commissioned. The first plant delivered is now fully operational, providing liquid helium for the magnet test hall. The first pre-series $1.8 \mathrm{~K}$ refrigeration unit has been successfully commissioned and approval for three series production has been given. The second pre-series $1.8 \mathrm{~K}$ refrigeration unit (from another company) is being commissioned and the production approval of the three remaining series units is awaiting final reception of the prototype.

The installation of the vertical cryogenic lines in the shafts is underway together with the cryogenic interconnection boxes at tunnel level. The installation of the critical cryogenic distribution line which distributes helium to the magnets, performs quench recovery and supplies the 15 mbar pumping pressure for the production of superfluid helium has started (Fig. 6).

\section{VACUUM}

The high intensity beams in the LHC will deposit heat into the cryogenic surface surrounding the beam through a number of effects, including image currents (up to about $0.8 \mathrm{~W} / \mathrm{m}$ ) and synchrotron radiation $(0.6 \mathrm{~W} / \mathrm{m})$. These heat loads cannot be taken at $1.9 \mathrm{~K}$ and will be intercepted by a beam screen fitted inside the magnet cold bore and cooled by circulation of supercritical helium between $5 \mathrm{~K}$ and $20 \mathrm{~K}$. Gas desorbed by the synchrotron radiation cannot be efficiently cryo-pumped by the screen at this 


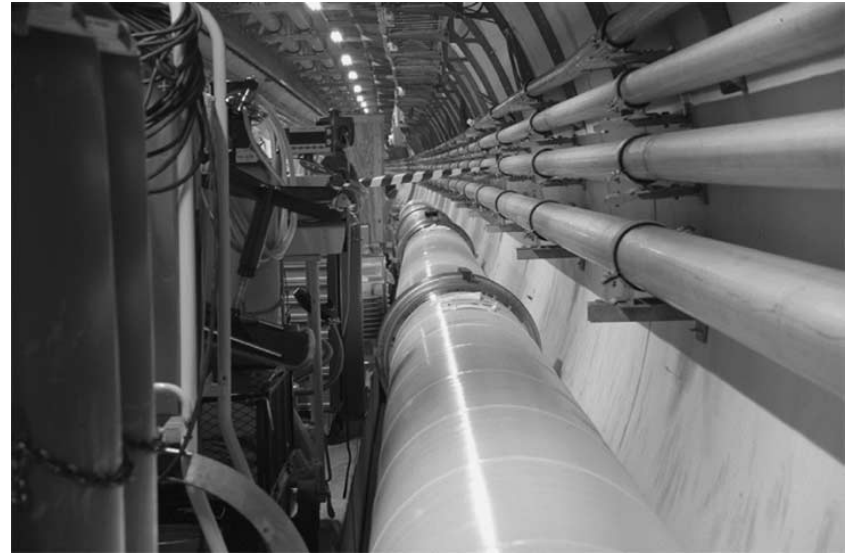

Fig. 6. Installation of the cryogenic distribution line (QRL) in the tunnel.

high temperature. In order to avoid a catastrophic pressure rise, the screen is punched with small holes over about $2 \%$ of its surface so that the cold bore of the magnets at $1.9 \mathrm{~K}$ can pump away the gas while being protected from the heat source. Heat can also be produced by inelastic scattering of protons with the residual gas molecules. This cannot be intercepted by the screen and must be transported away by the superfluid helium.

Another effect that can result in considerable heat input into the cryogenic system and vacuum degradation is due to beam induced multipactoring by the electric field of successive bunches as first observed in the ISR. It arises through a resonant motion of secondary electrons bouncing back and forth between the walls of the beam screen. If the secondary electron yield is sufficiently large, this can lead to an exponential build-up, stimulating gas desorption and heating of the beam screen. This effect is known to limit the performance of the B-factories and has received much attention in recent years. It has now been clearly observed in the SPS for LHC beam conditions [7]. As the electron bombardment of the surface proceeds, it has a conditioning effect, reducing the secondary electron yield and cleaning the surface of the chamber.

Recent experiments at the SPS, where the conditions for the onset of electron cloud buildup are very similar to the LHC, have clearly demonstrated this cleaning effect. Fig. 7 shows a measurement of the vacuum pressure during a four day "scrubbing" run. A reduction of 4 orders of magnitude over the scrubbing period can be observed. This is accompanied by a reduction of the secondary emission coefficient of the surface (see Fig. 8).

Experimental results have been compared with multiparticle simulation of the process [8]. For example, in a dipole field the simulation code predicts that the electron bombardment should be concentrated in two stripes on the top and bottom of the vacuum chamber where the separation varies linearly with bunch intensity. Fig. 9 shows a measurement of this effect in the SPS together with the most recent simulation results. These results have also confirmed that there is no position of the pumping slots in the LHC beam screen free from electron bombardment, and has resulted in a modification its design.

The scrubbing of the SPS vacuum chamber, together with the impedance reduction program previously mentioned has allowed the SPS to accelerate the full LHC beam to $450 \mathrm{GeV}$.
Further experiments are planned to investigate the efficiency of the scrubbing process on cold surfaces similar to the situation in the LHC itself.

In the warm regions of the $\mathrm{LHC}$, the electron cloud effect can be suppressed by coating the chamber with a new nonevaporable getter (NEG) material developed at CERN [9]. This material (TiZrV) can be activated at $200^{\circ} \mathrm{C}$, a temperature lower than for conventional getters. Once activated, the secondary electron yield is very low and, as shown in SPS experiments, the electron cloud effect is suppressed. These chambers also contribute to the production of ultra high vacuum due to their ability to pump gas. Production of coated chambers has now started.

\section{ACCELERATING SYSTEM}

The Radio Frequency system operates at $400.8 \mathrm{MHz}$, the second harmonic of the SPS frequency. Each beam has a separate system necessitating an increase of the beam separation from 194 to $410 \mathrm{~mm}$. Eight single-cell superconducting cavities per beam operating at an average gradient of $5 \mathrm{MV} / \mathrm{m}$ provides a total voltage of $16 \mathrm{MV}$. The cavities are made from copper sputtered with a thin film of niobium. All cavities have been manufactured and are being assembled into four-cavity modules (Fig. 10). The first module has been tested at full power with all ancillaries. Some problems with the power couplers are being resolved. An additional $200.4 \mathrm{MHz}$ system for efficient capture of the beam from the SPS has been foreseen, but recent measurements of longitudinal emittance in the SPS after completion of the impedance reduction program have confirmed that the installation of these cavities can be deferred until the LHC runs at its ultimate intensity.

The transverse feedback system (100 MHz bandwidth) for controlling transverse coupled-bunch instabilities is being constructed in collaboration with JINR (Dubna).

\section{OTHER SYSTEMS}

The LHC requires more than 1700 power converters ranging from $13 \mathrm{kA}$ to $60 \mathrm{~A}$ with very demanding specifications concerning precision and stability. All prototypes have been successfully tested and orders for series production have been placed.

The production of specialized equipment for beam injection and extraction (kickers, septa, pulse forming networks, etc.) is well advanced. A beam has already been extracted from the SPS at $450 \mathrm{GeV}$.

The ensemble of beam instrumentation has now been defined and series production of beam monitors and associated electronics has started. The SPS is proving to be a very useful test bed for this equipment.

In order to provide clean conditions for the detectors and to protect the machine against beam loss, two long straight sections are devoted to collimation. The design of these collimators is particularly demanding if they are to cope with all conceivable fault conditions. The technical design choice has recently been finalized and prototype construction is underway. 


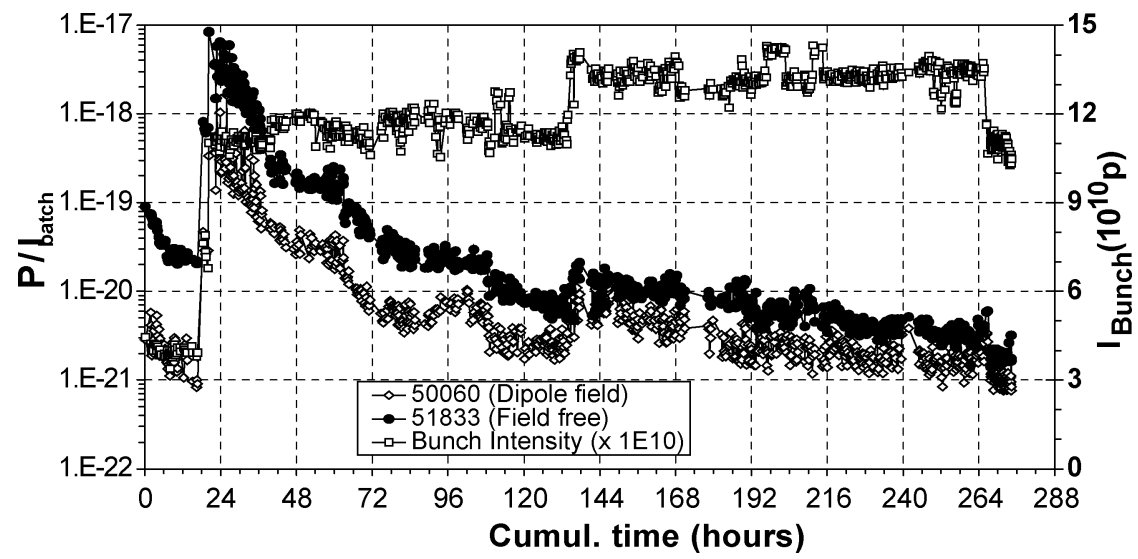

Fig. 7. Evolution of vacuum pressure with time during the SPS "scrubbing" run.

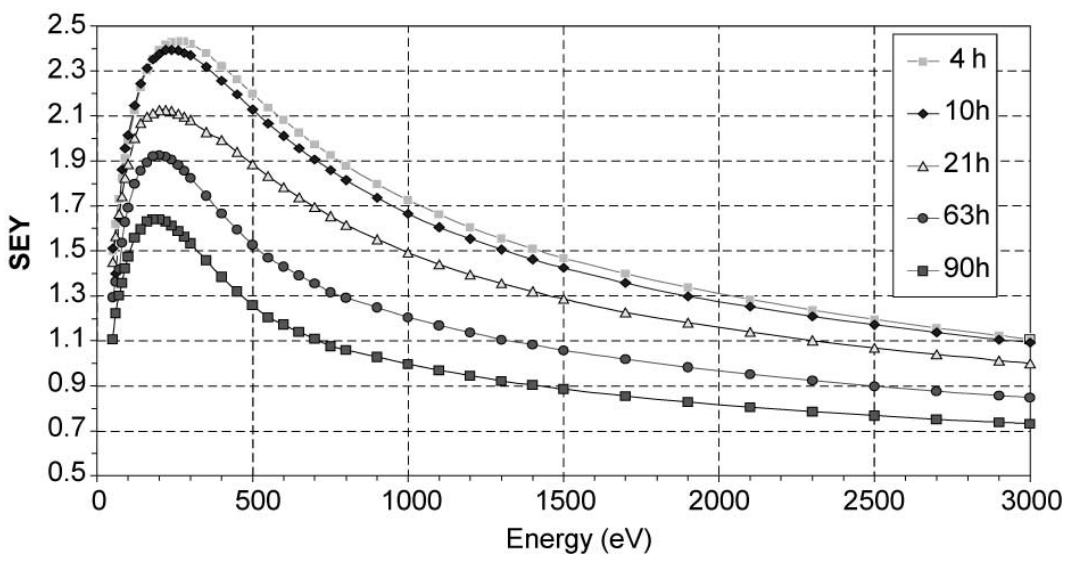

Fig. 8. Reduction of the secondary emission yield on the SPS vacuum chamber during the scrubbing process.

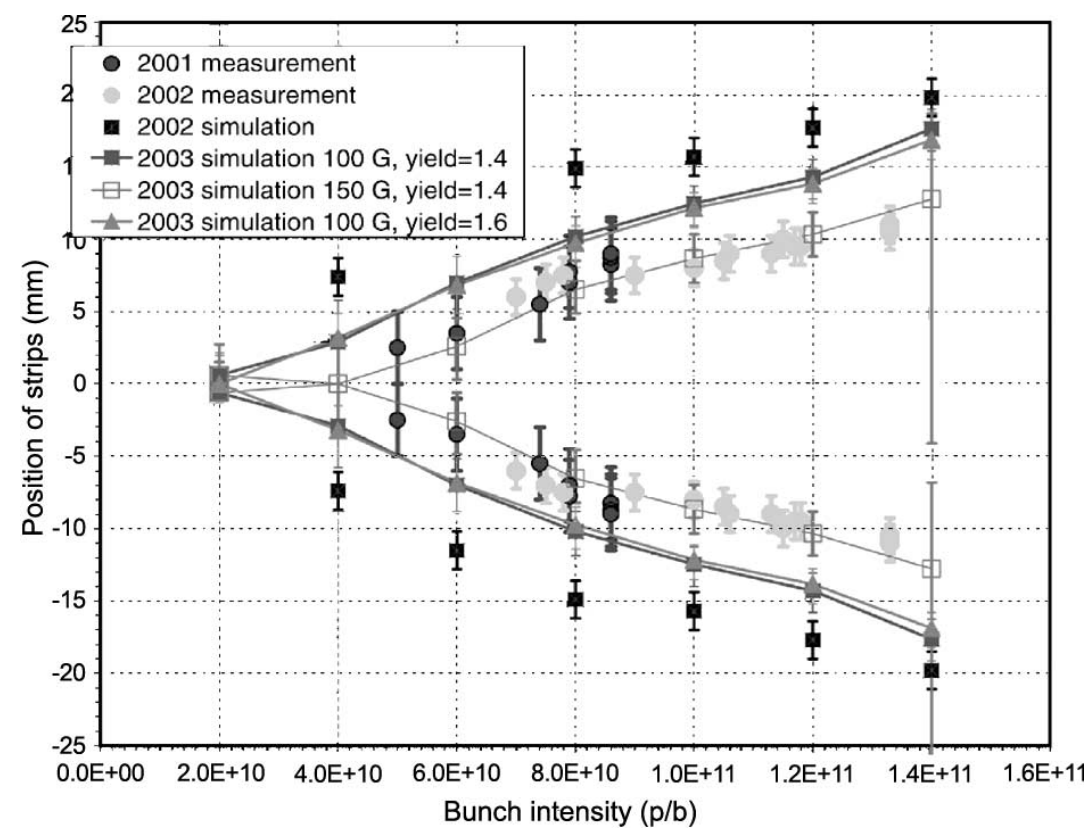

Fig. 9. Distance between the two strips as a function of intensity compared with simulation results.

\section{Civil CONSTRUCTION}

The civil engineering work consists of three main packages concerning the LHC machine and the experimental areas for
ATLAS and CMS respectively. For the machine, the work is distributed geographically, both for surface buildings housing the large cryogenic plants and in the underground areas. The most important underground work is the construction of two $2.5 \mathrm{~km}$ 


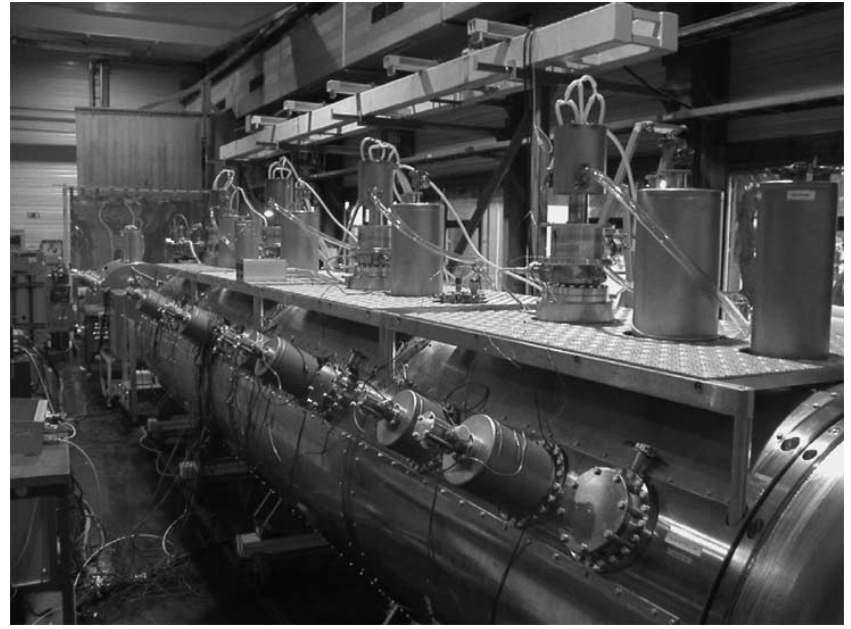

Fig. 10. A four-cell cavity module complete with couplers.

long tunnels housing the transfer lines from the SPS to the LHC. This work is now complete and installation of tunnel infrastructure is now underway. The ATLAS cavern has been delivered to the experimental collaboration and detector installation has started. The CMS cavern will be completed by summer 2004 . In the meantime, assembly of the CMS detector is proceeding in the surface building.

\section{SCHEDULE}

The original schedule, made in 1996, foresaw ring closure in summer 2005 and a short pilot run before a three month shutdown to finish the installation of the detectors. Since then, a number of factors, in particular the delay in LEP dismantling and the late delivery of the experimental caverns, has necessitated a revision of the schedule in collaboration with the exper- iment spokes-persons. According to the revised schedule, the ring will be closed by the end of 2006, with the first beam injected in spring 2007. The first physics run will take place in the second half of 2007. An important intermediate milestone will be in April 2006, when a beam will be injected at Point 8 and transported around the first octant to Point 7.

\section{CONCLUSION}

The LHC machine is now well into its installation phase. A realistic schedule has been set which is consistent with the observed ramp up of production of the most critical components. The LHC project team is fully committed to meeting this schedule and providing a first physics run in the second half of 2007.

\section{REFERENCES}

[1] R. Garoby, S. Hancock, and J. L. Vallet, "Demonstration of bunch triple splitting in the CERN PS," in Proc. 7th EPAC, Vienna, Austria, 2000, pp. 529-551.

[2] T. Bohl, T. Linnecar, and E. Shaposhnikova, "Impedance reduction in the CERN SPS as seen from longitudinal beam measurements," in Proc. 8th EPAC, Paris, France, 2002, pp. 1146-1148.

[3] E. Todesco et al., "Steering Field Quality in the Main LHC Dipoles,", this conference.

[4] L. Rossi, "Superconducting Magnets for the LHC Main Lattice,", this conference.

[5] J. Lucas et al., "Design, Assembly and Mechanical Behavior of the Preseries MQM-Type Cold Masses for the LHC Insertion Quadrupoles,", this conference.

[6] M. Allitt et al., "Production Status of the LHC Superconducting Corrector Magnets,", this conference.

[7] M. Jimenez et al., "Electron cloud studies and analyzes at SPS for LHC type beams," in 2003 PAC, Portland, USA, May 12-16, 2003.

[8] F. Zimmermann, "A Simulation Study of Electron Cloud Instability and Beam-Induced Multipactoring in the LHC,", CERN LHC Project Report 95, 1997.

[9] C. Benvenuti et al., "Vacuum properties of TiTrV nonevaporable getter films," Vacuum, vol. 60, p. 57, 2001. 